\title{
Oscillating and rotating sine-Gordon system
}

\section{Olsen, O. H.; Samuelsen, Mogens Rugholm}

\section{Published in:}

Physical Review B

Link to article, DOI:

10.1103/PhysRevB.33.595

Publication date:

1986

Document Version

Publisher's PDF, also known as Version of record

Link back to DTU Orbit

Citation (APA):

Olsen, O. H., \& Samuelsen, M. R. (1986). Oscillating and rotating sine-Gordon system. Physical Review B, 33(1), 595-598. https://doi.org/10.1103/PhysRevB.33.595

\section{General rights}

Copyright and moral rights for the publications made accessible in the public portal are retained by the authors and/or other copyright owners and it is a condition of accessing publications that users recognise and abide by the legal requirements associated with these rights.

- Users may download and print one copy of any publication from the public portal for the purpose of private study or research.

- You may not further distribute the material or use it for any profit-making activity or commercial gain

- You may freely distribute the URL identifying the publication in the public portal

If you believe that this document breaches copyright please contact us providing details, and we will remove access to the work immediately and investigate your claim 


\title{
Oscillating and rotating sine-Gordon system
}

\author{
O. H. Olsen \\ Systems Engineering, Niro Atomizer, Gladsaxevej 305, DK-2860 Soeborg, Denmark \\ M. R. Samuelsen \\ Physics Laboratory I, The Technical University of Denmark, \\ DK-2800 Lyngby, Denmark \\ (Received 31 January 1985)
}

\begin{abstract}
The interaction between a $2 \pi$ kink and the background or vacuum is investigated in the pure sineGordon system. For an oscillating background (i.e., the $k=0$ part of the phonon spectrum) the $2 \pi$ kink oscillates, while for increasing or decreasing vacuum two phenomena have been observed, depending on the rate of change of the vacuum. For small rates a parametric excitation of standing waves is found, and for larger rates the system linearizes. In the case of oscillating vacuum a perturbation approach explains the behavior perfectly, while for small rates of increasing vacuum the system reduces to a Mathieu equation explaining the excitation.
\end{abstract}

\section{INTRODUCTION}

There has recently been great interest in how a $2 \pi$-kink (soliton) solution to the sine-Gordon equation moves when perturbing terms are added to the sine-Gordon equation. ${ }^{1-4}$

The sine-Gordon equation, for instance, governs the $2 \pi$ kink ("fluxon") motion in a long and narrow Josephson tunnel junction and the perturbing terms represent losses, bias current, and applied microwaves. ${ }^{5}$

It turns out that the motion of the $2 \pi$ kink to a large extent depends on how the $2 \pi$ kink and especially its background initially is started. ${ }^{2,3}$ The differences in the motions can be understood as a result of an interaction between the $2 \pi$ kink and the motion of the background. ${ }^{2}$ If losses are present these differences in the $2 \pi$-kink motions due to the initial conditions rapidly die out. ${ }^{2}$

In this paper we will investigate this coupling between the $2 \pi$ kink and the background by considering the pure sineGordon equation. We consider the two different types of motion for the background: oscillation and monotonic increase/decrease (rotation). The former case has been examined in Ref. 1(c) and was referred to as a "screw effect." The aim of that investigation was to discuss the initial behavior of a $2 \pi \mathrm{kink}$, i.e., for times of, say, $t \leqslant 1$. The present analysis, which was first presented in Ref. 2, explains in a simple way the influence of the initial value of the vacuum on the $2 \pi$-kink dynamics. Further, this analysis holds for large times, say, $t=10-15$.

In Sec. II we outline the perturbation theory developed in Ref. 2. The results can be used to describe the interaction between the $2 \pi$-kink solution and the oscillating background in the pure sine-Gordon (SG) system. Comparisons between results from this theory and numerical experiments are performed in Sec. III. In Sec. IV the "rotating" background is considered. For small values of the angular velocity we find a parametric excitation of standing waves, while for large values of the angular velocity the equation linearizes. Finally, in Sec. V we comment and summarize the paper.

\section{PERTURBATION THEORY}

The equation in question is the pure sine-Gordon equation (in normalized form):

$$
\phi_{x x}-\phi_{t t}=\sin \phi \text {, }
$$

where the field variable is $\phi$. In the Josephson-junction case it is the macroscopic space- and time-dependent phase difference between the two superconductors. ${ }^{5}$ The space variable $x$ is normalized to the Josephson penetration depth $\lambda_{J}$ and the time variable $t$ to the reciprocal plasma frequency $\omega_{0}^{-1}$. $^{2}$

We seek a solution to Eq. (1), where $\phi(x= \pm \infty)$ is independent on $x$ and where we have a localized $2 \pi$ kink. The analysis in Ref. 2 is based on the assumption that the vacuum part of the solution satisfies the pendulum equation

$$
-\phi_{t t}^{\mathrm{vac}}=\sin \phi^{\mathrm{vac}} \text {. }
$$

The equation of motion for the $2 \pi$ kink is then found by calculating the total wave momentum resulting in the following equation:

$$
P_{K}(t)=2 \pi \phi_{t}^{\mathrm{vac}}(t)+\text { const } .
$$

Here $P_{K}(t)$ is the momentum of the $2 \pi$ kink and const is a constant of integration determined by the initial condition

$$
\text { const }=P_{K}(0)-2 \pi \phi_{t}^{\mathrm{vac}}(0) \text {. }
$$

Up until now no approximation has been made. The approximation we use here is to put the $2 \pi$-kink part of the momentum $P_{K}$ equal to the pure sine-Gordon soliton value

$$
P_{K}=8 u \gamma(u), \quad \gamma(u)=\left(1-u^{2}\right)^{-1 / 2}
$$

where $u$ is the $2 \pi$-kink normalized velocity. This method has proved to give excellent results with perturbation terms in the sine-Gordon equation. ${ }^{2}$ 


\section{OSCILLATING BACKGROUND}

In this section we will compare analytical solutions to numerical solutions of the same initial value problem. In all cases considered here we choose the initial $2 \pi$-kink velocity to be zero, i.e., $P_{K}(0)$ in Eq. (4) equal to zero. Then Eq. (3) becomes

$$
P_{K}(t)=2 \pi\left[\phi_{t}^{\mathrm{vac}}(t)-\phi_{t}^{\mathrm{vac}}(0)\right] .
$$

Then the analytical problem is reduced to solve Eq. (2) for the initial values imposed upon $\phi_{t}^{\text {vac }}(t)$ and use Eq. (5) to find the $2 \pi$-kink velocity

$$
u=\frac{P_{K}(t)}{\sqrt{64+P_{K}(t)^{2}}} .
$$

Equation (1) is solved numerically by the method of characteristics and the initial condition is

$$
\phi(x, 0)=4 \tan ^{-1} \exp (x)+\phi^{\mathrm{vac}}(0),
$$

and

$$
\phi_{t}(x, 0)=\phi_{t}^{\mathrm{vac}}(0) .
$$

The velocity of the $2 \pi$ kink is numerically determined $a^{2}$

$$
u(t)=-\frac{\phi_{t}^{\max }(t)-\phi_{t}^{\mathrm{vac}}(t)}{\phi_{x}^{\max }(t)},
$$

where $\phi_{t}^{\max }(t)$ and $\phi_{x}^{\max }(t)$ are the values of $\phi_{t}$ and $\phi_{x}$ at the time $t$ and at the point where the derivative $\phi_{x}$ has its maximum.

The oscillating solution to Eq. (2) can be expressed in terms of Jacobian elliptic functions ${ }^{6}$

$$
\phi^{\operatorname{vac}}(t)=2 \sin ^{-1}\left[\sin \left(\frac{1}{2} \phi_{m}\right) \operatorname{sgn}\left(t-t_{0}\right)\right] .
$$

Here $\phi_{m}$ is the maximum value for $\phi^{\mathrm{vac}}(t)$ in the oscillation and $t=t_{0}$ is the time where

$$
\phi^{\mathrm{vac}}\left(t_{0}\right)=0 \text {, }
$$

and where

$$
\phi_{t}^{\mathrm{vac}}\left(t_{0}\right)=2 \sin \left(\frac{1}{2} \phi_{m}\right) \equiv \omega=2 \sqrt{m},
$$

which define $\omega$, the angular velocity of the "pendulum in the bottom."

In Fig. 1 we illustrate the kind of motion there can be with an oscillating background. The $2 \pi$ kink is started on the middle of a 30 long line with $\phi^{\mathrm{vac}}(0)=\pi / 4$ and $\phi_{t}^{\text {vac }}(0)=0$ [in Eq. (10) $\phi_{m}=\pi / 4$ and $t_{0}=K(m)$, the complete elliptic integral of first kind, which is a quarter of the period of the oscillation]. The motion is followed for 21 time units; it is seen how the background oscillation forces the $2 \pi$ kink to oscillate. The small amount of radiation produced by the oscillation does not influence the basic dynamics of the $2 \pi$ kink-see Fig. 2.

In Fig. 2 we compare the analytical solution [Eq. (10) into Eq. (6) into Eq. (7)] with the numerically determined velocities [Eq. (9)]. In Fig. 2(a) the initial condition is $t_{0}=0$ and $\sin \left(\frac{1}{2} \phi_{m}\right)=\omega / 2\left[\phi^{\mathrm{vac}}(0)=0\right.$ and $\left.\phi_{t}^{\mathrm{vac}}(0)=\omega\right]$ for $\omega=0.125,0.25$, and 0.5 . For these small $\omega$ values Eq. (10) is very close to the pure trigonometric variation $[\sin \phi \simeq \phi$ in Eq. (2)]. The agreement is seen to be good. In Fig. 2(b) the initial conditions are $t_{0}=K \quad\left[\phi^{\mathrm{vac}}(0)=\phi_{m} \quad\right.$ and $\left.\phi_{t}^{\text {vac }}(0)=0\right]$ for $\phi_{m}=0.125,0.25$, and 0.5. Again Eq. (10)

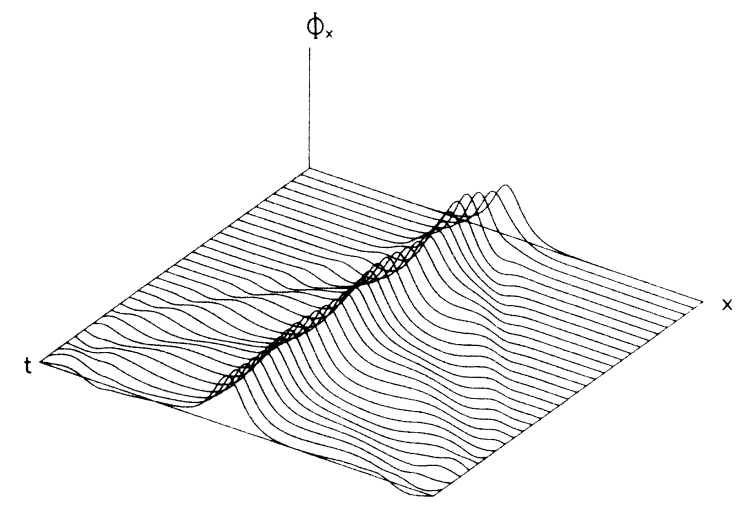

FIG. 1. Illustration of the interaction between the $2 \pi$ kink and the background. The results are displayed in terms of $\phi_{x}$ for $0 \leqslant x \leqslant 30$ and $0 \leqslant t \leqslant 21$. At $t=0, \phi^{\operatorname{vac}}(0)=\pi / 4$ and $\phi_{t}^{\operatorname{vac}}(0)=0$. The $2 \pi$ kink oscillates while it creates radiation.
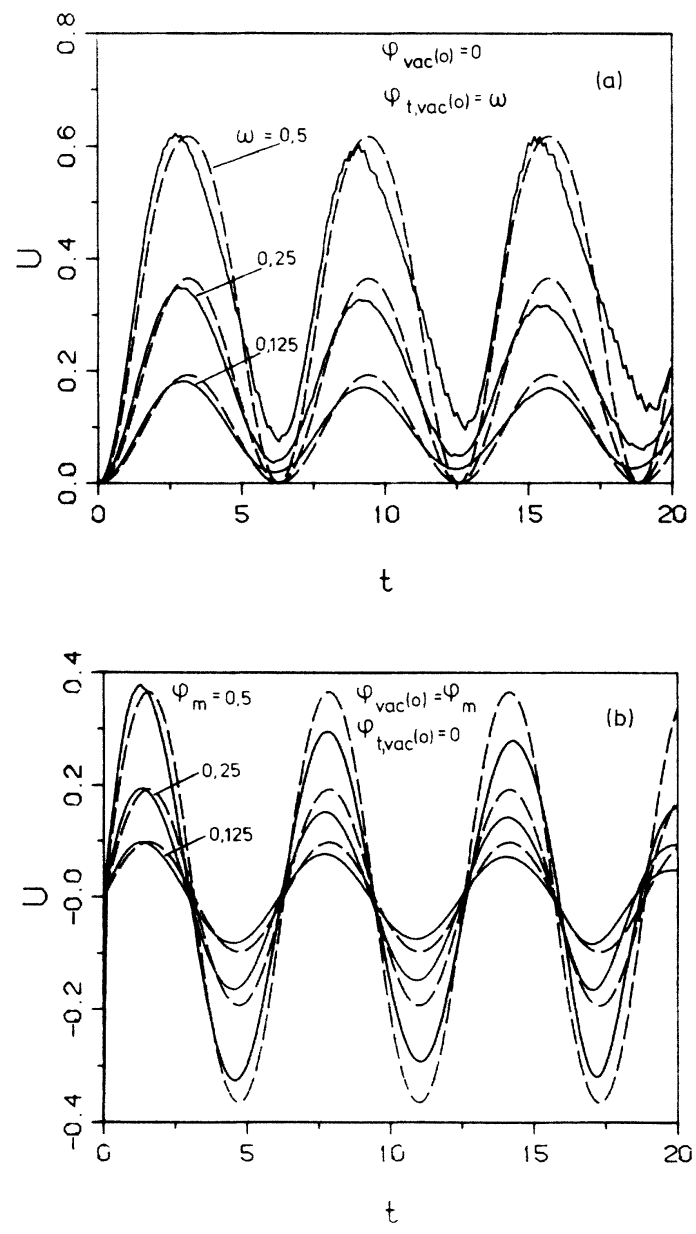

FIG. 2. Comparison between analysis and numerical experiments like the one in Fig. 1; broken line is the analysis. In (a) $\phi^{\mathrm{vac}}(0)=0$ and $\phi_{t}^{\mathrm{vac}}(0)=\omega$; for $\phi^{\mathrm{vac}}(t)<<1$ the analytical expression for $u$ is given by Eq. (7), where $P_{k}(t)=2 \pi \omega(1-\cos t)$. In (b) $\phi^{\text {vac }}=\phi_{m}$ and $\phi_{t}^{\text {vac }}(0)=0$; for $\phi^{\mathrm{vac}}(t) \ll 1$ the analytical expression for $u$ is again given by Eq. (7), where $P_{k}(t)=2 \pi \phi_{m} \sin t$. The agreement between analysis and numerical experiment is good. 


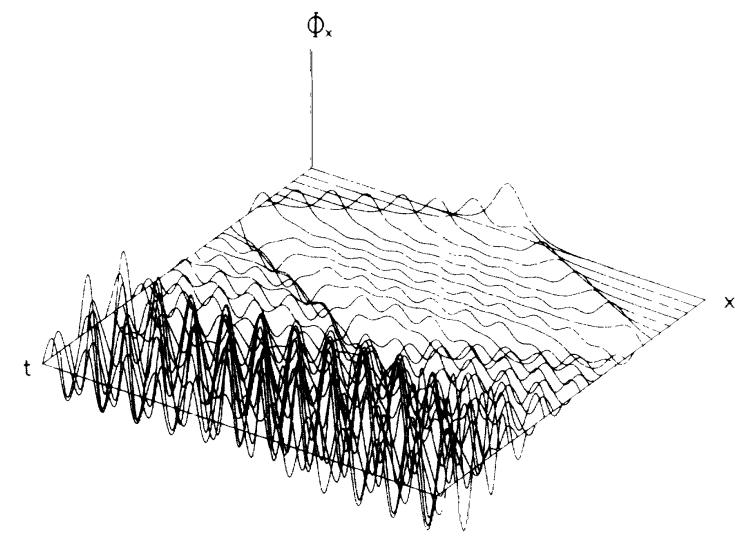

FIG. 3. Illustration of parametric excitation of standing waves. The $2 \pi$ kink is initially placed in the middle of a 30 long line; $\phi^{\text {vac }}(0)=0, \phi_{t}^{\text {vac }}(0)=5,0 \leqslant t \leqslant 60$. The $2 \pi$ kink splits into $\pi$ kinks moving in opposite directions. Eventually, the parametric excitation occurs, which is independent of the presence of the $2 \pi$ kink. The frequency of the excitated waves is half the average velocity of the background.

is close to a trigonometric variation and the agreement is good.

\section{ROTATING BACKGROUND}

If the angular velocity of the pendulum in the bottom $\omega$ exceeds two, the pendula start to rotate [see Eq. (12)] and the solution to Eq. (2) is

$$
\phi^{\mathrm{vac}}(t)=2 \cos ^{-1}\left\{\mathrm{cn}\left[\left(t-t_{0}\right) / \sqrt{m}\right]\right\},
$$

and for $\phi_{t}^{\mathrm{vac}}(t)$,

$$
\phi_{t}^{\mathrm{vac}}(t)=2 \mathrm{dn}\left[\left(t-t_{0}\right) / \sqrt{m}\right] / \sqrt{m},
$$

especially in the bottom,

$$
\phi_{t}^{\mathrm{vac}}\left(t_{0}\right) \equiv \omega=\frac{2}{\sqrt{m}} \text {. }
$$

In Figs. 3 and 4 we illustrate the kinds of motions there can be with a rotating background. Again the $2 \pi$ kink is started in the middle of a 30 long line with $\phi^{\mathrm{vac}}(0)=0$ and $\phi_{t}^{\text {vac }}(0)=\omega\left(t_{0}=0\right)$ for $\omega=5$ (Fig. 3) and 10 (Fig. 4). The motion is followed for 60 time units. It is seen that the $2 \pi$ kink splits into two kinks moving in opposite directions with velocity one. Therefore the perturbation method used until now based on a localized $2 \pi$ kink cannot be used. The result in Fig. 4 can be understood directly from Eq. (1), since $\phi$ is rapidly rotating (the critical value was $\omega=2$ ) averaging the $\sin \phi$ term almost to zero. Then we are left with a pure linear dispersion-free wave equation. Therefore the $2 \pi$ kink splits into two $\pi$ kinks moving with velocities \pm 1 . Due to the boundary condition $\phi_{x}=0$, the $\pi$ kink is reflected as a $-\pi$ kink and vice versa. The beginning of the development in Fig. 3 can be qualitatively understood in the same way.

The later development in Fig. 3, the apparently very irregular waves, would develop without the initial presence of a $2 \pi$ kink. It is a parametric excitation of standing waves possible for a certain combination of the length $l$ of the line

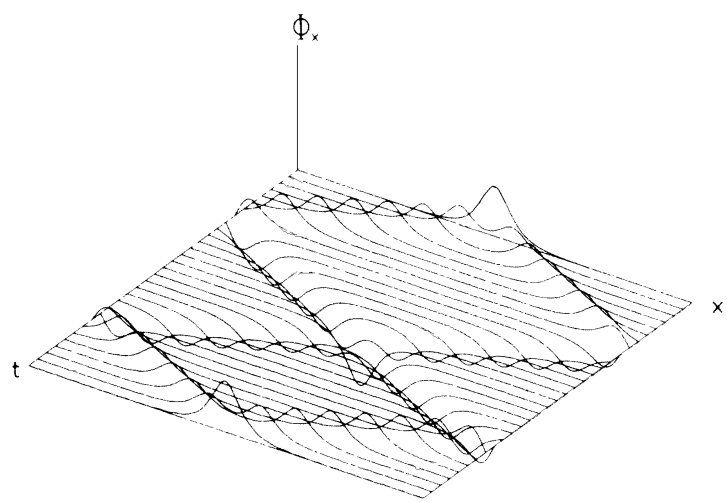

FIG. 4. The same initial conditions as in Fig. 3, except $\phi_{t}^{\text {vac }}(0)=10$. Due to the higher value of $\phi_{t}^{\text {vac }}$ the parametric excitation disappears-the system linearizes.

and the initial angular velocity $\omega$.

Let us consider an initial situation without a $2 \pi$ kink and Eq. (13) as the initial condition and write the solution to Eq. (1) as

$$
\phi(x, t)=\phi^{\operatorname{vac}}(t)+\delta \phi(x, t),
$$

where $\delta \phi(x, t)$ is a small deviation from $\phi_{\text {vac }}(t)$. Imposing the boundary condition

$$
\phi_{x}(0, t)=\phi_{x}(l, t)=0 \text {, }
$$

we try a solution of the form

$$
\delta \phi=\cos k x \cdot \delta \phi(t)
$$

Introducing Eqs. (16) and (18) into Eq. (1) and linearizing with respect to $\delta \phi$, we get

$$
\delta \phi_{\|}+\left[k^{2}+\cos \phi^{\mathrm{vac}}(t)\right] \delta \phi=0 .
$$

From Eq. (17) the wave number $k$ has to fulfill

$$
k=\frac{n \pi}{l} \text {, }
$$

where $n$ is an integer.

This is a Hill-type equation, which yields nonsmall solutions for $\delta \phi(t)$ if certain conditions are fulfilled. If we approximate $\phi^{\text {vac }}(t)$ by

$$
\phi^{\mathrm{vac}}(t)=\omega_{\mathrm{corr}} \cdot\left(t-t_{0}\right)
$$

where $\omega_{\text {corr }}$ is the average of $\phi_{t}^{\text {vac }}(t)$; i.e.,

$$
\omega_{\text {corr }}=\frac{\pi}{2 K(m)} \cdot \frac{2}{\sqrt{m}}=\frac{\pi}{2 K\left[(2 / \omega)^{2}\right]} \cdot \omega .
$$

Then Eq. (19) becomes a Mathieu equation ${ }^{6}\left(t_{0}=0\right)$,

$$
\delta \phi_{t}+\left(k^{2}+\cos \omega_{\text {corr }} t\right) \delta \phi=0,
$$

or, in the usual notation, ${ }^{6}$

$$
\delta \phi_{z z}+[a-2 q \cos (2 z)] \delta \phi=0 .
$$




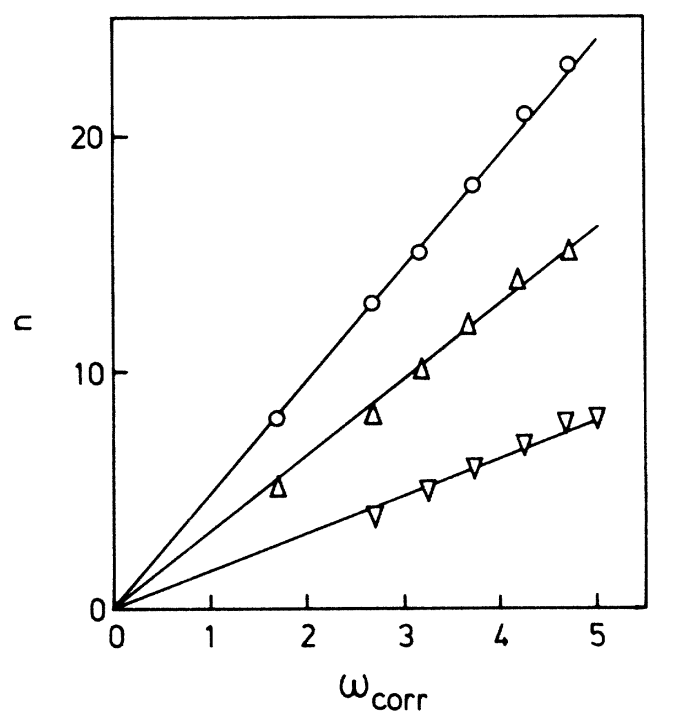

FIG. 5. Comparison between the analysis [Eq. (24)] (full line) and numerical experiments like the one in Fig. 3 . The length $l$ of the line is, from the top, $l=30,20,10$. For $\omega_{\text {corr }} \sum 5$ the parametric excitation disappears.

where $a=\left(2 k / \omega_{\text {corr }}\right)^{2}$ and $q=2 / \omega_{\text {corr }}^{2}$ Now, harmonic solutions to Eqs. (23) with frequency $\omega_{\text {corr }} / 2$ exist if $a=1$, or $\omega_{\text {corr }} / 2-k$ and $q$ is not too small. The frequency in Fig. 3 is 2.4 , while $\omega_{\text {corr }}=4.7$, indicating that the arguments above are valid. From Eq. (20) it follows that

$$
n=k l / \pi=\left(\omega_{\text {corr }} / 2 \pi\right) l
$$

In Fig. 5 we have displayed results from numerical experiments like the one in Fig. 3. The full curves are Eq. (24), while the points are the measured $n$; from the top we have $l=30,20,10$. The agreement between the analysis and the numerical experiments is good. When $\omega_{\text {corr }} \geq 5$ the parametric excitation disappears ( $q$ is too small).

\section{CONCLUSION}

In the present paper we have investigated the interaction between a $2 \pi$ kink and the background or vacuum. For oscillating background the $2 \pi$ kink oscillates. This behavior can easily be explained by means of a perturbation approach. The agreement between the results obtained from the perturbation approach and numerical experiments is good.

For increasing or decreasing (rotating) background, two different phenomena are found, depending on the rate of change of the background. For small rates $\omega_{\text {corr }} \leqslant 5$ a parametric excitation of standing waves is observed, while for larger rates the system linearizes. The parametric excitation can be explained by means of an ansatz, which reduces the system to a Mathieu equation. In certain parameter ranges this equation possesses harmonic solutions of frequency equal to $\omega_{\text {corr }} / 2$, in agreement with the numerical experiments.
1 (a) J. C. Fernandez, M. J. Gambaudo, S. Gauthier, and G. Reinisch, Phys. Rev. Lett. 46, 753 (1981); (b) G. Reinisch and J. C. Fernandez, Phys. Rev. B 24, 835 (1981); (c) 25, 7352 (1982); (d) 27, 5817 (1983).

${ }^{2}$ O. H. Olsen and M. R. Samuelsen, Phys. Rev. Lett. 48, 1569 (1982); Phys. Rev. B 28, 210 (1983).

3P. C. Dash, Phys. Rev. Lett. 51, 2155 (1983); Phys. Lett. 104A,
309 (1984).

${ }^{4}$ A. M. Kosevich and Yu. S. Kivshar, Phys. Lett. 98A, 237 (1983); and in Nonlinear and Turbulent Processes in Physics, edited by R. Z. Sagdeev (Harwood Academic, New York, 1984), p. 1401.

${ }^{5}$ N. F. Pedersen and D. Welner, Phys. Rev. B 29, 2551 (1984).

${ }^{6}$ Handbook of Mathematical Functions, edited by M. Abramowitz and I. A. Stegun (Dover, New York, 1970). 